\title{
EL ESTATUS TAXONÓMICO DE MYLABRIS (MESOSULCATA) THAMII KOCHER, 1963, UN ENDEMISMO DEL SUR DE MARRUECOS (COLEOPTERA, MELOIDAE)
}

\author{
J. L. Ruiz (*)
}

\begin{abstract}
RESUMEN
En el presente trabajo, se propone el estatus taxonómico específico de Mylabris (Mesosulcata) thamii Kocher, 1963, originalmente descrita como subespecie de Mylabris hirtipennis Raffray, 1873. Se aporta una redescripción de la especie, resaltando sus principales caracteres morfológicos externos y genitales (morfología de la cabeza, ojos y antenas, punteado de la cabeza y pronoto, estructura del edeago). Se discuten los rasgos diagnósticos y respectivas distribuciones geográficas de M. thamii y M. hirtipennis. Asimismo, se exponen los caracteres diagnósticos diferenciales entre las tres especies que actualmente constituyen el subgénero Mesosulcata Pardo Alcaide, 1950 (M. hirtipennis, M. thamii y M. hieracii Graëlls, 1849) y se ofrece una clave de determinación de las mismas.
\end{abstract}

Palabras clave: Coleoptera, Meloidae, Mylabris, Mesosulcata, Mylabris (Mesosulcata) thamii, taxonomía, distribución, Marruecos.

\begin{abstract}
The taxonomic status of Mylabris (Mesosulcata) thamii Kocher, 1963, a southern Moroccan endemism (Coleoptera, Meloidae)

In this paper, the taxonomic specific status of Mylabris (Mesosulcata) thamii Kocher, 1963, originally described as subspecies of Mylabris hirtipennis Raffray, 1873, is proposed. A redescription of the species is given, emphasizing the principal external morphological and genital traits (head, eyes and antennae morphology, pronotum and head puncture, aedeagus structure). Diagnostic characters and geographic range of $M$. thamii and $M$. hirtipennis are discussed. Also, distinctive traits among the species of subgenus Mesosulcata Pardo Alcaide, 1950 (M. thamii, M. hirtipennis and M. hieracii Graëlls, 1849) are presented in a diagnostic key.
\end{abstract}

Key words: Coleoptera, Meloidae, Mylabris, Mesosulcata, Mylabris (Mesosulcata) thamii, taxonomy, distribution, Morocco.

\section{Introducción}

El subgénero Mesosulcata (especie tipo: Mylabris hieracii Graëlls, 1849), integrado en el género Mylabris Fabricius, 1775, fue creado por Pardo Alcaide (1950), originalmente como "sec- ción" del subgénero Mylabris (sensu Pardo Alcaide, 1950), para acomodar a Mylabris hieracii Graëlls, 1849, especie ibérica bien definida por caracteres relativos a la morfología externa y a la conformación del edeago. Más tarde, este autor (Pardo Alcaide, 1954a, 1954b) incluye en dicha

* Avda. Madrid, 4, $1^{\circ}$ izq. 51001 Ceuta. España. 
sección a la especie magrebí Mylabris hirtipennis Raffray, 1873, que comparte los rasgos distintivos del subgénero: placa mesosternal sin escudo central $\mathrm{y}$ con dos surcos longitudinales divergentes hacia atrás, lóbulo medio del edeago de tipo isoharpagae (sensu Pardo Alcaide, 1948) y lóbulos apicales de los parámeros con una pilosidad muy corta en sus áreas lateroventrales (Pardo Alcaide, 1950, 1954a, 1954b, 1964; Bologna, 1991).

Paralelamente, Kuzin (1954) crea el subgénero Glaucabris, al que adscribe las dos especies citadas. Bologna (1991, pág. 204) propone la sinonimia entre ambos subgéneros, correspondiéndole la prioridad nomenclatural a Mesosulcata, el cual debe ser considerado subgénero válido (Bologna, 1991, 1994).

Según lo expuesto, el subgénero Mesosulcata agrupa, hasta el momento, únicamente a dos especies (Bologna, 1991, 1994), Mylabris hieracii y Mylabris hirtipennis; la primera de ellas de distribución normediterránea occidental, presente en la mayor parte de la Península Ibérica ("Guadarrama", loc. typ.) y Francia sudoccidental (e. g. Graëlls, 1849, 1851; Marseul, 1870, 1872; Górriz y Muñoz, 1882; López Neyra, 1914; Soumakov, 1915; Borchmann, 1917; Mader, 1927; Fuente, 1933; Pardo Alcaide, 1950; Valladares, 1984; Bologna, 1991; Bologna y Moragues, 1993), habiendo sido citada del Atlas marroquí por Heyden (1887), muy posiblemente por confusión con M. hirtipennis (Bologna, 1991); la segunda, de distribución magrebínica occidental, ocupa Marruecos y Argelia (e.g. Raffray, 1873; Escherich, 1899; Escalera, 1909, 1914; Soumakov 1915; Borchmann, 1917; Pardo Alcaide, 1954a, 1954b; Kocher, 1956; Bologna, 1991), y también fue señalada por Soumakov (1934) de Algeciras (provincia de Cádiz, España), probablemente confundida con la especie anterior (Bologna, 1991).

Por otro lado, Kocher (1963), a partir de tres ejemplares procedentes de la localidad presahariana marroquí de Hassi-Bou-Guejouf (bajo Oued Draa, Thami Lachheb leg.), describe Mylabris hirtipennis thamii, resaltando la merionalidad de la localización de esta subespecie, ya que $M$. hirtipennis nunca había sido hallada al sur del Alto Atlas. Desde su descripción, y a excepción de la coetánea Addenda al catálogo de los coleópteros de Marruecos publicada por el propio Kocher (1964), este taxon ha pasado desapercibido, considerándose en la actualidad integrado el subgénero Mesosulcata por las dos especies antes señaladas (Bologna, 1991, 1994).

En el presente trabajo, sobre la base de diversos caracteres morfológicos externos y genitales, se propone el estatus taxonómico específico de $M$. thamii Kocher, 1963, especie que queda encuadrada en el subgénero Mesosulcata. Se ofrece una descripción detallada de la especie, puesto que la original es sumamente escueta y no alude a ciertos rasgos de importancia diagnóstica. Asimismo, se discuten los caracteres diagnósticos de $M$. thamii frente a los de $M$. hirtipennis, especie a la cual es más cercana morfológica y geográficamente. Por último, se indican los principales rasgos diferenciales entre $M$. hieracii y $M$. thamii y se ofrece una clave de identificación de las especies del subgénero Mesosulcata.

\section{Material y métodos}

Se han estudiado un total de 9 ejemplares de $M$. thamii y 37 de $M$. hirtipennis, material que se encuentra conservado en el Museo Nacional de Ciencias Naturales de Madrid, el Institut Royal des Sciences Naturelles de Belgique, Bruselas, así como en la colección del autor. La lista del material examinado se expone en el Apéndice I.

El estudio morfológico de los ejemplares se realizó con material en seco, mediante observación con estereomicroscopio. Las genitalias, una vez extraídas a ejemplares conservados en seco previamente reblandecidos, se montaron en cartulinas con resina D.M.H.F., las cuales se pincharon con su respectivo ejemplar. Las medidas se efectuaron con micrómetro acoplado a uno de los oculares y los dibujos con cámara clara. La nomenclatura de las diferentes partes de la genitalia masculina se basa en la expresada por Pardo Alcaide (1948, 1950, 1954b) y Bologna (1991).

\section{Resultados}

La descripción original de Mylabris hirtipennis thamii es muy breve (Kocher, 1963, pág. 36) y se refiere exclusivamente a los caracteres que su autor considera discriminantes con respecto a la subespecie nominal. En concreto, sólo hace referencia a su tamaño menor $(6,5-7 \mathrm{~mm})$, región anterior del cuerpo más brillante y puntos negros de los élitros hundidos ["comme chez hemprichi Klug" (sic)].

El estudio de nuevos ejemplares adscribibles a esta subespecie, procedentes de Tizi-n-Tinifift (Agdz), nos revela la presencia de un mayor número de rasgos diagnósticos válidos, constantes en los nueve especímenes estudiados, que avalarían el estatus específico que se propone, constituyendo 

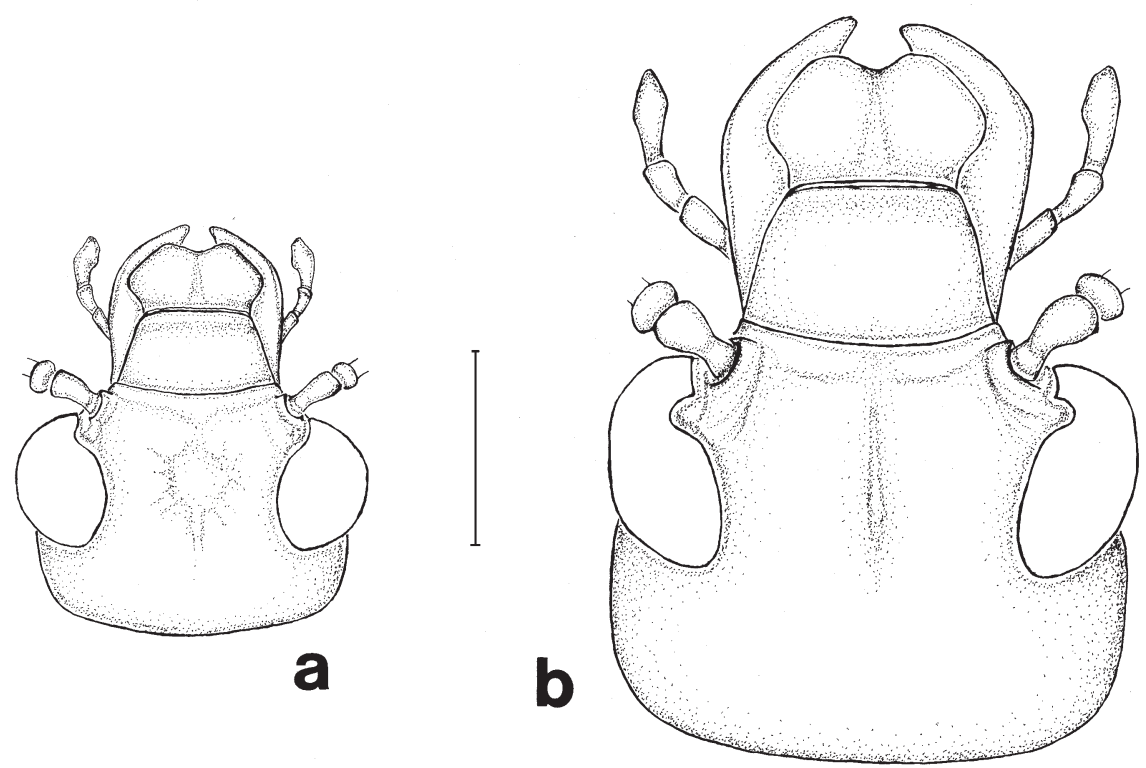

Fig. 1.- Cabeza (esquemática), en visión dorsal, de: a) Mylabris (Mesosulcata) thamii (Tizi-n-Tinifift, Anti Atlas); b) Mylabris (Mesosulcata) hirtipennis (Imlil, Alto Atlas). Escala $=1 \mathrm{~mm}$.

Fig. 1.- Head (schematic), in dorsal view, of: a) Mylabris thamii (Tizi-n-Tinifift); b) Mylabris hirtipennis (Imlil, High Atlas). Scale $=1 \mathrm{~mm}$.

por tanto la tercera especie conocida del subgénero Mesosulcata Pardo Alcaide, 1950. La localidad de captura de los individuos examinados se sitúa en la cabecera del río Draa (Oued Draa), curso fluvial en cuya cuenca baja, a unos $390 \mathrm{~km}$ al suroeste, se ubica la localidad típica de $M$. thamii.

Al objeto de caracterizar morfológicamente a $M$. thamii, se ofrece a continuación una redescripción de la especie, poniendo de manifiesto sus principales rasgos diagnósticos y la variabilidad observada.

Mylabris (Mesosulcata) thamii Kocher, 1963 stat. nov., comb. nov.

Mylabris hirtipennis s.sp. thamii Kocher, 1963. Bull. Soc. Sci. Nat. Phys. Maroc, 43: 36

REDESCRIPCIÓN: Longitud: 7,4-9,8 mm [media= $8,42 \mathrm{~mm} ; \mathrm{n}=9$; no obstante, el rango de tamaños debe ser más amplio, pues Kocher (1963) lo sitúa entre 6,5-7 mm]. Anchura máxima, situada a nivel del tercer cuarto elitral: 2,84-3,65 mm (media= $3,09 \mathrm{~mm}, \mathrm{n}=9$ ).

Coloración general del cuerpo negra, de aspecto muy brillante, tornándose castaño oscura en el extremo de los apéndices. Élitros amarillo anaran- jados, con tres series transversas de manchas puntiformes negras. Pubescencia negra, larga y erizada sobre el cuerpo, más corta en los élitros y tumbada en el cuarto distal de los mismos.

Cabeza (Figs. 1a, 2a) con tegumento negro y brillante, truncada por detrás; sienes cortas, aproximadamente la mitad de largas que el diámetro transversal del ojo en visión lateral [relación "anchura del ojo/longitud de la sien" ("c/d", ver Fig. 2a) entre 1,7-2,3; media $=1,86 ; n=9]$, ligeramente convergentes hacia atrás y muy redondeadas en los ángulos posteriores; frente plana, con una zona lisa deprimida en el disco de contorno irregular y límites mal definidos, de cuyo margen posterior parte hacia atrás una arruga irregular subcareniforme; sutura frontal marcada y débilmente arqueada; epistoma transverso, subtrapezoidal, unas 1,7 veces más ancho que largo; labro transverso, entre 1,9-2,3 veces más ancho que largo (media $=2,1 ; n=9$ ), deprimido en el disco y con un surco central que partiendo de la base lo recorre en casi toda su longitud, lados ampliamente redondeados y con la escotadura central del margen anterior amplia y poco profunda; mandíbulas de aproximadamente igual longitud que los ojos, con sus márgenes externos rectos en sus $2 / 3$ anteriores y curva- 

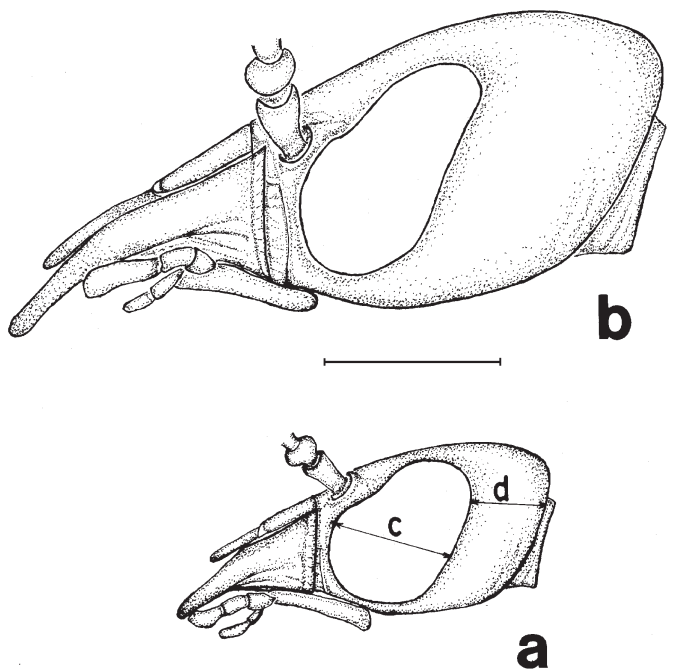

Fig. 2.- Cabeza (esquemática), en visión lateral, de: a) Mylabris thamii (Tizi-n-Tinifift); b) Mylabris hirtipennis (Imlil, Alto Atlas). Escala $=1 \mathrm{~mm}$.

Fig. 2.- Head (schematic), in lateral view, of: a) Mylabris thamii (Tizi-n-Tinifift); b) Mylabris hirtipennis (Imlil, High Atlas). Scale $=1 \mathrm{~mm}$.

dos en el tercio distal; maxilas y palpos no modificados. Ojos anchos y muy globosos, muy particulares, poco escotados en la región contigua a las inserciones antenares, imprimiéndole un aspecto general subredondeado al conjunto de la cabeza. Punteado muy irregular, constituido por puntos gruesos pero poco profundos, muy difusos, visibles solamente en los laterales de la frente, en las sienes y algo en el vértex, conformándose en la mayor parte de la región frontal y vértex en arrugas transversas $\mathrm{u}$ oblicuas, que ofrecen un aspecto general subvermiculado. Punteado del epistoma constituido por puntos netos de menor tamaño que los de la frente, separados entre una y dos veces su diámetro, los del labro a su vez algo menores que los anteriores y más dispersos. Pilosidad negra, corta y erecta en la región anterior de la cabeza, más larga hacia atrás y los lados, y casi inexistente en la zona central de la frente (posiblemente debido a pérdida por rozamiento).

Antenas (Fig. 3a) de once artejos, relativamente cortas, de aspecto mazudo, engrosadas de la mitad hacia delante, zona esta última donde los antenómeros se muestran fuertemente comprimidos, sobre todo los tres últimos; antenómero I aproximadamente dos veces más largo que el segundo, robus-

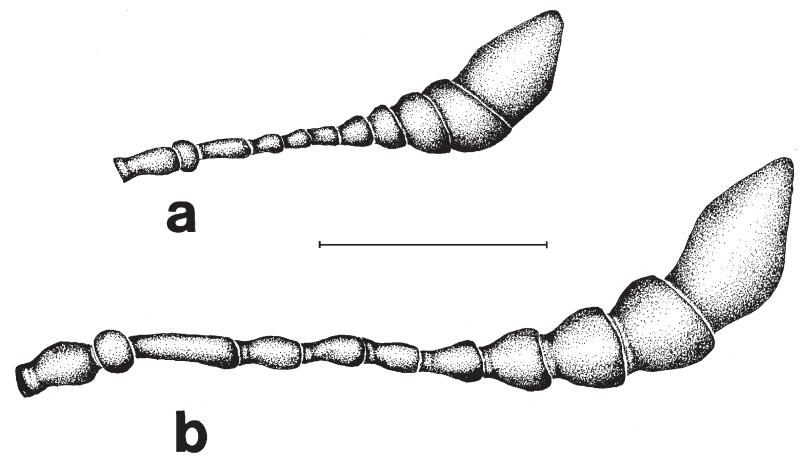

Fig. 3.- Antena de: a) Mylabris thamii (Tizi-n-Tinifift); b) Mylabris hirtipennis (Imlil, Alto Atlas). Escala $=1 \mathrm{~mm}$.

Fig. 3.- Antenna of: a) Mylabris thamii (Tizi-n-Tinifift); b) Mylabris hirtipennis (Imlil, High Atlas). Scale $=1 \mathrm{~mm}$.

to, aunque poco engrosado a partir del estrechamiento basal; II corto y subgloboso; III subparalelo, estrecho, de longitud aproximada como el I más la mitad del II; IV al VI subiguales en longitud y forma, casi cilíndricos, algo ensanchados en sus extremos; VII de longitud similar al anterior pero más ensanchado en su zona distal; VIII trapezoidal, un poco más ancho que largo en su extremidad; IX de forma muy similar al anterior, muy ensanchado en su extremo, donde es una vez y media más ancho que largo; X subtrapezoidal, muy comprimido contra los dos artejos contiguos, algo más de una vez y media más ancho que largo; XI un poco más estrecho que el anterior, subparalelo hasta la mitad y a partir de aquí estrechándose cónicamente hasta el extremo. Antenómeros I y II con pilosidad larga y erecta, del III al VIII con pelos muy cortos e inclinados, y del IX al XI cortísimos y tendidos.

Pronoto entre 1,13-1,22 veces más ancho que largo (media $=1,17, \mathrm{n}=9$ ), deprimido hacia delante en visión lateral y truncado en el margen anterior, con una ligera sinuosidad centro-basal por delante del escutelo, presentando tres depresiones transversas de límites difusos pero bien perceptibles, una anterior, otra discal y la tercera basal justo por delante de la sinuosidad; lados, en visión dorsal, subparalelos en sus $2 / 3$ posteriores y convergentes en el tercio anterior. Punteado pronotal irregular, difuso, muy semejante al de la cabeza y sustituido en su mayor parte por arrugas, concentrándose sobre todo en el tercio anterior y entre la depresión basal y la discal, esta última con escasos puntos y rodeada de arrugas irregulares más o menos longi- 
tudinales; áreas declive laterales con muy escasos puntos. Pilosidad larga, inclinada hacia delante en la mitad anterior y más o menos erecta en la mitad posterior, escasa en el centro y más densa hacia los lados, siguiendo en general el mismo patrón de distribución que los puntos en los que se inserta.

Escutelo negro, pequeño, hemielíptico, anchamente redondeado por detrás e impunteado.

Mesosterno sin escudo central y con dos surcos longitudinales marcados divergentes hacia atrás, característico de los integrantes del subgénero Mesosulcata (Pardo Alcaide, 1950, 1954b; Bologna, 1991), lados ligeramente prolongados hacia delante; vértice posterior poco prolongado; superficie lisa y brillante, sólo con algunos puntos pequeños dotados de pelos alargados en los lados y junto a los márgenes laterales. Mesopleuras con el borde libre anterior finamente aquillado y con la foseta o escotadura central pequeña y bien marcada.

Élitros amarillo anaranjados, con tres series oblicuas de dos manchas negras puntiformes y hundidas $(2,2,2)$, de las cuales la mancha externa se dirige hacia atrás. Punteado constituido por puntos relativamente pequeños pero netos y apretados, escasos en el cuarto anterior y casi inexistentes en la región humeral. Pilosidad negra, la mitad de larga que la pronotal, inclinada hacia delante en la mitad anterior, donde en algunos ejemplares es muy escasa, debido probablemente a pérdida por rozamiento, perpendiculares a la superficie elitral en el tercer cuarto e inclinada hacia atrás en el cuarto posterior.

Región ventral del cuerpo negra, con pubescencia alargada. Último esternito abdominal visible escotado en su margen posterior en el macho.

Patas de aspecto grácil, con pilosidad normal, corta e inclinada. Tibias anteriores en la cara interna con una banda longitudinal de pelos amarillo blanquecinos muy cortos y densos, en la cara externa algunos pelos negros muy largos, portando en su extremo apical externo una muy pequeña expansión digitiforme en los machos. Protarsómeros con un cepillito de cortos e hirsutos pelos blanquecinos, al igual que los cuatro primeros mesotarsómeros. Espinas metatibiales cortas, estrechas e iguales.

Edeago (Figs. 4a, 4b, 4c) con los parámeros relativamente cortos, los lóbulos distales poco alargados, levantados y apenas curvados, portando escasos y cortísimos pelos amarillentos en sus áreas lateroventrales. La región ventral inmediatamente anterior a los lóbulos paramerales, en visión lateral, describe un arco ligeramente cóncavo, casi recto. Falobase corta y ancha. Lóbulo medio con dos
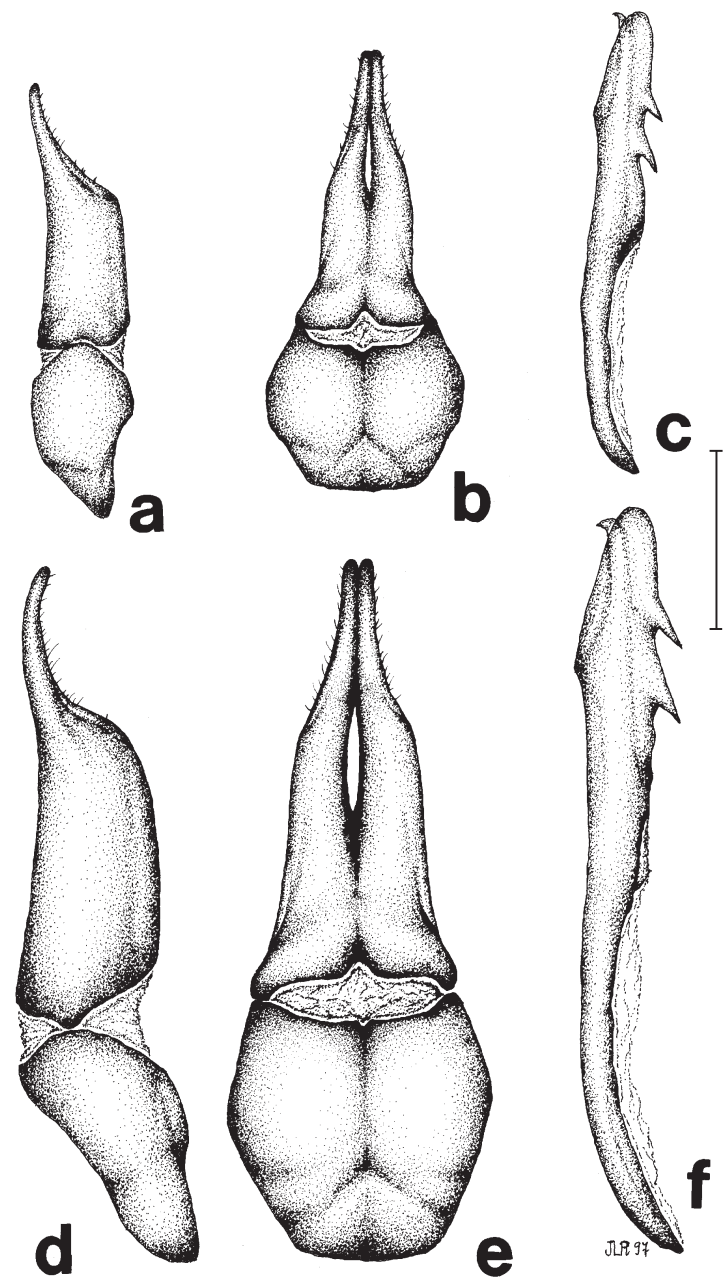

Fig. 4.- Mylabris thamii (Tizi-n-Tinifift): a) parámeros y falobase en visión lateral; b) parámeros y falobase en visión dorsal; c) lóbulo medio en visión lateral. Mylabris hirtipennis (Imlil, Alto Atlas): d) parámeros y falobase en visión lateral; e) parámeros y falobase en visión dorsal; f) lóbulo medio en visión lateral. Escala $=0,5 \mathrm{~mm}$.

Fig. 4.- Mylabris thamii (Tizi-n-Tinifift): a) parameres and phallobasis, lateral view; b) parameres and phallobasis, dorsal view; c) median lobe, lateral view. Mylabris hirtipennis (Imlil, High Atlas): d) parameres and phallobasis, lateral view; e) parameres and phallobasis, dorsal view; f) median lobe, lateral view. Scale $=0,5 \mathrm{~mm}$.

pequeños dientes subiguales netamente separados del ápice, ambos dispuestos en un similar ángulo de inclinación.

La hembra es similar al macho, pero diferenciada de éste por presentar las expansiones digitiformes del extremo externo de las tibias anteriores 
bastante más largas y desarrolladas, al igual que la pilosidad de la cara externa de las mismas, la pilosidad elitral de mayor longitud y el último esternito abdominal visible no escotado en su margen posterior. Asimismo, en los tres ejemplares hembra examinados los ojos se muestran ligeramente más globosos.

VARIABILIDAD: Los nueve especímenes examinados presentan unos rangos de variabilidad muy exiguos respecto a caracteres discriminantes, afectando de forma claramente perceptible únicamente al tamaño corporal, anchura relativa de los ojos y tenues diferencias en la densidad del punteado y pilosidad de la cabeza y pronoto. El diseño cromático elitral se muestra muy estable, con muy pequeñas variaciones en el diámetro de las manchas negras puntiformes, que en ningún momento llegan a unirse entre sí ni a desaparecer.

DisTRIBUCIÓN Y NOTAS AUTOECOLÓGICAS: Hasta el momento, $M$. thamii sólo se conoce de dos localidades, ambas en el sur de Marruecos: Hassi-BouGuejouf (locus typicus) y Tizi-n-Tinifift. La primera de ellas se localiza en la cuenca baja del Draa, extremo suroeste de Marruecos, en las estribaciones suroccidentales del Anti Atlas. La región es de una aridez extrema; así, la cercana localidad de Assa $(35 \mathrm{~km}$ al este) registra una precipitación media anual de tan solo $68 \mathrm{~mm}$, encuadrándose en la zona ecoclimática hiperárida superior (Le Houerou, 1989). Desde el punto de vista geológico, la zona se enmarca en el dominio del Anti Atlas occidental, con rocas paeleozoicas afectadas por metamorfismo de alto grado y afloramientos de granitos hercinianos (Michard, 1976). El puerto de montaña de Tizi-n-Tinifift $(1600 \mathrm{~m})$ se encuentra situado en el extremo oriental de la cadena montañosa del Anti Atlas, casi a caballo entre ésta y el macizo del Djebel Sahrro, y a unos $8 \mathrm{~km}$ en línea recta de la localidad de Agdz, en el margen derecho de la cuenca alta del río Draa (Oued Draa). Al igual que la anterior, esta localidad presenta una marcada aridez; los datos climatológicos existentes se refieren a la cercana localidad de Agdz (1100 m), con una precipitación media de tan solo $108 \mathrm{~mm}$ anuales, encuadrándose la región en la zona ecoclimática árida inferior (Le Houerou, 1989). Geológicamente pertenece a la unidad estructural del Anti Atlas, con litología constituida fundamentalmente por cuarcitas del paleozoico (Michard, 1976).

Los individuos estudiados fueron colectados en las horas centrales de la jornada en un día soleado, sobre compuestas (Asteraceae) de bajo porte e inflorescencias amarillas (Vela y Bastazo, com. pers., 1996), a principios del mes de abril. Kocher (1963) no hace referencia a las circunstancias de captura de los tres ejemplares que conforman la serie típica, señalando únicamente la época de captura, entre finales de febrero y principios de marzo. En vista a los escasos datos fenológicos existentes, el período de actividad imaginal de $M$. thamii se sitúa entre finales de invierno y principios de primavera. La fenología precoz de la especie ha de ser interpretada como respuesta a las condiciones de elevada aridez y temprana floración reinantes en las regiones donde habita, como ocurre en otros Mylabrini de distribución sahariana y presahariana, por ejemplo algunas especies del género Croscherichia Pardo Alcaide, 1950 (Bologna y Coco, 1991).

\section{Discusión}

Mylabris thamii es una especie afín a Mylabris hirtipennis, con la que comparte los caracteres diagnósticos del subgénero Mesosulcata en el que ambas se encuadran, además de un semejante diseño cromático elitral y corporal. Sin embargo, muestra una serie de características distintivas evidentes y constantes en los nueve ejemplares examinados, relativos tanto a la morfología externa como a la armadura genital masculina, que apoyan el estatus taxonómico específico propuesto. Tales caracteres no fueron ponderados por Kocher (1963) en la descripción de la subespecie; no obstante, este autor apuntaba la posibilidad de que pudiera tratarse de una especie vicariante de $M$. hirtipennis.

Mylabris thamii se segrega fácilmente de $M$. hirtipennis por la conjunción de los siguientes caracteres: contorno de la cabeza de aspecto más redondeado y ancho (Figs. 1a, 1b); sienes significativamente más cortas y menos rectas (Figs. 2a, 2b), mostrando una relación "anchura del ojo/longitud de la sien" (c/d, ver Fig. 2a) comprendida entre 1,7 y 2,3 (media= 1,86, n=9), mientras que en $M$. hirtipennis oscila entre 0,65 y 1 (media $=0,88 ; n=17)$; ojos más anchos, globosos y salientes; labro más ancho (relación "anchura/longitud" entre 1,9 y 2,3; media $=2,1 ; \mathrm{n}=9$; variando en $M$. hirtipennis entre $1,6$ y 1,$75 ;$ media $=1,7 ; n=17)$; antenas de aspecto más mazudo, con los antenómeros a partir del VIII bastante más anchos y comprimidos (Figs. 3a, 3b), sobre todo el IX y X, algo más de una vez y media más anchos que largos, siendo en $M$. hirtipennis aproximadamente igual o un poco más anchos en 
su extremo que largos; punteado de la cabeza y pronoto superficial, difuso y poco denso, sustituido en las áreas discales de la frente y pronoto por pequeñas arrugas transversas, muy diferente al de $M$. hirtipennis, donde es fuerte, profundo y denso, nada difuso; pronoto con la depresión central más extensa y prácticamente lisa, ésta en $M$. hirtipennis se encuentra enteramente punteada. El edeago también presenta diferencias notables (Fig. 4): en $M$. thamii los lóbulos paramerales son más cortos y levantados, apenas curvados; la falobase es más corta y ancha; y la región ventral inmediatamente anterior a los lóbulos paramerales describe una línea casi recta o ligeramente cóncava (en visión lateral), mientras que en $M$. hirtipennis esta zona es manifiestamente convexa y los lóbulos paramerales curvados. Por último, el tamaño corporal de $M$. thamii $[7,4-9,8 \mathrm{~mm}$; media $=8,42 ; \mathrm{n}=9$; entre $6,5-7$ mm según Kocher (1963)] se sitúa en el límite inferior de tamaño expresado para M. hirtipennis [8-20 mm, según Pardo Alcaide (1954b)], e incluso por debajo de éste.

Todos los ejemplares de $M$. hirtipennis examinados (ver Apéndice I) comparten un patrón morfológico similar respecto a los caracteres expuestos en la discriminación específica, aunque están sujetos a cierto grado de variación individual y poblacional. Dicha variabilidad afecta principalmente al tamaño [10,8-16,9 mm, media $=14,5 ; n=17$; según Pardo Alcaide (1954b) entre 8-16 mm]; diseño elitral, con las manchas puntiformes de mayor o menor tamaño, desapareciendo en ciertos casos alguna de las internas y/o externas, o en el caso opuesto, llegando a unirse las de la serie posterior entre sí y a su vez con la externa de la serie central (ejemplar de Ketama, Rif); punteado de pronoto y cabeza con ligeras variaciones en cuanto a su densidad, pero siempre apretado, neto y fuertemente impreso; cuatro últimos antenómeros a veces algo más transversos, particularmente en algunos especímenes del Atlas Medio, en este sentido, Escalera (1909) incluye a $M$. hirtipennis en un grupo de especies caracterizado por presentar sus antenas con "maza más aparente y los artejos de ésta bastante o muy apretados", pero sin llegar en ningún momento al morfotipo antenar de M. thamii; y por último, lóbulos paramerales más o menos alargados, pero siempre algo curvados.

Por otra parte, M. hieracii, distribuida por la Península Ibérica y Francia sudoccidental (e. g. Pardo Alcaide, 1950; Bologna, 1991), se separa sin dificultad de $M$. thamii por su tamaño mayor (10-20 $\mathrm{mm})$; antenas comparativamente más largas, ensanchadas progresivamente hacia el ápice, pero sin aspecto mazudo y artejos no comprimidos; tegumento corporal siempre con reflejos azulado-metálicos; élitros con una estrecha lúnula apical negra; y punteado de la cabeza y pronoto profundo y denso, además de una genitalia diferente (Pardo Alcaide, 1950, págs. 64-65, figs. 7-8; Bologna, 1991, págs. 215-217, fig. 79). Las tres especies que constituyen el subgénero Mesosulcata pueden separarse con la siguiente clave:

1. Tegumento corporal azulado metálico o negro con reflejos azulados. Élitros con una estrecha lúnula apical negra. Antenas progresivamente ensanchadas hacia el ápice pero no mazudas, con artejos nada comprimidos

M. (Mesosulcata) hieracii

1'. Tegumento corporal negro, sin reflejos metálicos. Élitros sin lúnula apical negra. Antenas de aspecto más o menos mazudo, con artejos antenales en parte comprimidos .... 2

2. Antenas muy mazudas, con artejos IX y X más de una vez y media más ancho que largos y muy comprimidos. Ojos muy globosos y salientes, sienes cortas (anchura del ojo/longitud de la sien=1,7-2,3). Cabeza y pronoto con punteado superficial, difuso y poco denso, sustituido en áreas discales por arruguitas transversas. Pilosidad del pronoto escasa. Lóbulos paramerales del edeago apenas curvados. Tamaño menor (6,5-9,8 $\mathrm{mm})$

M. (Mesosulcata) thamii

2'. Antenas menos mazudas, con artejos IX y X apenas algo más anchos que largos y menos comprimidos. Ojos menos globosos y sienes más largas (relación anchura del ojo/longitud de la sien $=0,65-1,0)$. Cabeza y pronoto con punteado fuertemente impreso y denso. Pilosidad del pronoto densa. Lóbulos paramerales del edeago curvados. Tamaño mayor $(8-20 \mathrm{~mm}) \quad$............... M. (Mesosulcata) hirtipennis

Mylabris hirtipennis fue descrita a partir de ejemplares procedentes del bosque de Boghari ("Forêt de Boghari", loc. typ.), Argelia (Raffray, 1873), país donde ocupa las regiones montañosas septentrionales de influencia mediterránea (Chobaut, 1902; Cros, 1939). Su área de distribución en Marruecos comprende los principales núcleos montañosos y algunas estaciones costeras (Pardo Alcaide, 1954b). En concreto, en el ámbito geográfico marroquí ha sido citada de las siguientes localidades: Rif: Isaguen (Pardo Alcaide, 1954b), Talass-n-Tane, Talamske (Kocher, 1964); Medio Atlas: Azrou (Soumakov, 1934), Ifrane (Kocher, 1955); Alto Atlas y sus estribaciones occidentales: Arround, Reraia, Amismiz, Djebel Amsitten (Soumakov, 1934), Tizi-n-Tacherat [cercanías del puerto de "Tisi-Tacherat", en el valle superior del Reraia, localidad de la que Escherich (1899) describió Zonabris kabylica, sinonimizada con M. hirtipennis por Chobaut (1902)], SidiRahal, Essaouira (antigua Mogador), Marraquech (Escalera, 1909, 1914; Kocher, 1956), Tizi-nTachdirt (3300 m) y Tinzerht (Alluaud, 1926; Cros, 
1939; Kocher, 1956). La localidad costera de Safi, citada por Soumakov $(1934)$ y considerada excéntrica por Kocher (1956), queda confirmada (ver Apéndice I). Por último, Chobaut (1902) añade la localidad de "Oued Aouerne", que sitúa en el "Atlas marroquí", sin especificar si se trata del Alto o Medio Atlas.

A pesar de las reservas impuestas por los escasos datos corológicos disponibles, $M$. hirtipennis y M. thamii parecen presentar áreas de distribución alopátricas. Así, M. hirtipennis ocupa en Marruecos el norte, centro y suroeste, no sobrepasando hacia el sur el Alto Atlas en el interior y la localidad de Essaouira en la franja costera atlántica. Mylabris thamii se comporta como un elemento faunístico propio del macizo del Anti Atlas y regiones áridas presaharianas circundantes, incluidos los tramos medio y bajo del Oued Draa. Por el momento no se conocen áreas de contacto entre poblaciones de ambas especies, que hipotéticamente podrían localizarse en la región de Ouerzazate, intermedia entre el Alto Atlas y el extremo nororiental del Anti Atlas y cercana a la localidad de Agdz.

El subgénero Mesosulcata presenta un rango geográfico relativamente estrecho, circunscrito a la región mediterránea occidental, con límites septentrional y meridional en el sudoeste de Francia $(M$. hieracii) y regiones norsaharianas marroquíes $(M$. thamii) respectivamente. La distribución de Mesosulcata le imprime cierta singularidad frente a otros subgéneros de Mylabris de ámbito paleártico occidental, cuyas áreas de distribución son comparativamente más extensas (e.g. Bologna, 1991), constituyendo el único de distribución exclusivamente ibero-magrebí. No obstante, presenta un amplio espectro ecológico, ocupando ambientes muy diversos, desde bosques montanos húmedos mediterráneos de Pinus sylvestris Linné (M. hieracii; Bologna, 1991) hasta regiones semiáridas en la Península Ibérica (Ruiz et al., 1994) y áridas en el sur de Marruecos (M. thamii). Igualmente, muestra un dilatado rango altitudinal, desde el nivel del mar hasta la alta montaña, con techo a los $3300 \mathrm{~m}$ en el Alto Atlas (M. hirtipennis). En este sentido, contrasta el relativamente estrecho area de distribución y la escasa diversidad específica alcanzada en el subgénero, sólo tres especies, con la variedad de hábitats que ocupa.

\section{AGRADECIMIENTOS}

A los Drs. José Miguel Vela y Gloria Bastazo por cedernos para su estudio los Meloidae por ellos colectados en Marruecos y ser los desencadenantes del presente trabajo. A Juan de Ferrer
Andreu por el apoyo bibliográfico prestado. A D. Francisco Delgado y D. Simón Chamorro, que nos proporcionaron información geográfica y geológica de Marruecos. Al Dr. José M. Ávila (Universidad de Granada), que puso a nuestra disposición los Mylabrini por él colectados en Marruecos. Al Dr. Mario García París (MNCN) por su inestimable ayuda en todo momento. A las Dras. Mercedes París, Carolina Martín e Isabel Izquierdo, que nos facilitaron el estudio de los Mylabris de la Colección Entomológica del Museo Nacional de Ciencias Naturales de Madrid y al Dr. Marcel Cludts por el préstamo de material del Institut Royal des Sciences Naturelles de Belgique.

\section{Referencias}

Alluaud, C., 1926. Compte rendu d'une mission zoologique dans le Maroc sud-oriental, avril-mai 1924. Bulletin de la Société des Sciences Naturelles du Maroc, 6: 12-28.

Bologna, M.A., 1991. Fauna d'Italia. XXVIII. Coleoptera Meloidae. Calderini. Bologna. 541 pp.

BolognA, M.A., 1994. I Meloidae della Grecia (Coleoptera). Fragmenta Entomologica, 25. Supplemento: 1-119.

BolognA, M.A. \& Coco, E., 1991. Revisione del genere Croscherichia Pardo Alcaide, 1950 (Coleoptera, Meloidae). Memorie della Società Entomologica Italiana, 69 [1990]: 97-180.

Bologna, M.A. \& Moragues, G., 1993. Zonitis fernancastroi Pardo Alcaide, espèce nouvelle pour la faune de France, et données faunistiques sur Zonitis nana Ragusa (Coleoptera, Meloidae). Nouvelle Revue d'Entomologie (Nouvelle série), 10(4): 369-373.

Borchmann, F., 1917. Pars 69. Meloidae, Cephaloidae. In: E. Schenkling, (ed.). Coleopterorum Catalogus auspiciis et auxilio W. Junk. Junk, Berlin. 208 pp.

Chobaut, A., 1902. Identité des Mylabris hirtipennis Raffr. et kabylica Esch. (Col.). Bulletin de la Société Entomologique de France, 1902: 164-165.

Cros, A., 1939. Les Meloides des possesions françaises de l'Afrique du Nord. (Étude biogéographique). Bulletin et Annales de la Société Royale Entomologique de Belgique, 79: 247-265.

EsCALERA, M.M. DE LA, 1909. Especies de Zonabris del SW de Marruecos. Boletín de la Real Sociedad Española de Historia Natural, 9: 244-249.

EsCALERA, M.M. DE LA, 1914. Los Coleópteros de Marruecos. Trabajos del Museo Nacional de Ciencias Naturales de Madrid. Serie zoológica, 11: 1-553.

Escherich, K., 1899. Zur Kenntniss der Coleopterengattung Zonabris Harold. Wiener Entomologische Zeitung, 18: 84-109.

Fuente, J.M. DE LA, 1933. Meloidae. In: Catálogo sistemático-geográfico de los Coleópteros observados en la Península ibérica, Pirineos propiamente dichos y 
Baleares (pars.). Boletín de la Sociedad Entomológica de España, 16: 18-49.

GóRRIZ Y MUÑOZ, R.J., 1882. Ensayo para la Monografía de los coleópteros Meloideos indígenas con aplicación a las ciencias médicas. Sanz y Navarro. Zaragoza. 201 pp.

GRAËLlS, M.M. DE LA P., 1849. (Description de Coléoptères nouveaux propes à la faune centrale de l'Espagne). Revue et Magasin de Zoologie: 620-622.

GRAËLLS, M.M. DE LA P., 1851. Description de quelques Coléoptères nouveaux de la faune centrale d'Espagne. Annales de la Société Entomologique de France, 9(2): 5-27.

Heyden, L. von, 1887. Bericht über die von Herren Prof. Dr. Freiherrn von Fritsch und Prof. Dr. Rein in Marocco und dem Atlas-gebirge gesammelten Käfer. Deutsche Entomologische Zeitschrift, 31: 433-448.

KocHER, L., 1955. Variation chez certains Mylabris marocains. Comptes rendus des séances mensuelles de la Société des Sciences Naturelles et Physiques du Maroc, 7: 127-128.

Kocher, L., 1956. Catalogue commenté des Coléoptères du Maroc. V. Hétéromères (Tenebrionides excepts). Travaux de l'Institut Scientifique Chérifien (série zoologie), 10: 1-107.

Kocher, L., 1963. Nouvelle recolte des Coléoptères dans le Bas Drâ (Sahara occidental marocain). Bulletin de la Société des Sciences Naturelles et Physiques du Maroc, 43: 35-37.

Kocher, L., 1964. Catalogue commenté des Coléoptères du Maroc. X. Addenda et Corrigenda. Travaux de l'Institut Scientifique Chérifien (série zoologie), 30: 1-200.

KuZIN, V.S., 1954. K posnanyju sistemy narybnikov (Coleoptera, Meloidae). Trudy Entomologischeskogo Obshchestva, 44: 336-379.

Le Houerou, H.N., 1989. Classification écoclimatique des zones arides (s.1.) de l'Afrique du Nord. Ecologia Mediterranea, 15(3-4): 95-144.

López Neyra, C.R., 1914. Claves dicotómicas para la determinación de los meloidos indígenas. Boletín de la Real Sociedad Española de Historia Natural, 14: 461-475.

Mader, L., 1927. Meloidae. In: Winkler, A. (ed.). Catalogus Coleopterorum Regionis Palaearcticae. II: 851-888. Winkler. Wien.

Marseul, S.A. DE, 1870. Monographie des Mylabrides d'Europe et des contrées limitrophes en Afrique et en Asie. L'Abeille, 7: 1-204.

Marseul, S.A. DE, 1872. Monographie des Mylabrides. Mémories de la Société Royale des Sciences de Liège: 363-662.

Michard, A., 1976. Eléments de Géologie marocaine. Notes et Mémories Service Géologique du Maroc, 252: $1-408$
Pardo Alcaide, A., 1948. Estudios sobre Meloidae. I. Acerca de la validez específica de Mylabris maculoso-punctata Grll., rosinae Escher. y pauper Escher. Eos, 24: 493-502.

PARdo Alcaide, A., 1950. Estudios sobre Meloidae. II. Los "Mylabrini" de la Península ibérica. Boletín de Patología Vegetal y Entomología Agrícola, 17 [1949]: 61-82.

PARdo AlcAide, A., 1954a. Estudios sobre Meloidae. IV. Sobre algunos Mylabris Fab. del occidente norteafricano. (Primera parte). Eos, 30: 331-344.

Pardo Alcaide, A., 1954b. Études sur les Meloidae. V. Les Mylabrini du Maroc et du Sahara occidental occidental espagnol (Col. Meloidae). Bulletin de la Société des Sciences Naturelles et Physiques du Maroc, 34: 55-88.

Pardo Alcaide, A., 1964. Études sur les Meloidae. XVII. Matériaux pour une révision des Mylabrini de 1'Afrique du Nord et du Moyen Orient (Coleoptera). Eos, 40: 529-544.

RAfFray, A., 1873. Second partie. In: Fairmaire, L. y Raffray, A., 1873. Coléoptères du Nord de l'Afrique. Revue et Magasin de Zoologie, 1873: 379-378.

Ruiz, J.L., SÁnchez-Piñero, F. \& Ávila, J.M., 1994. Faunística y corología de los Meloidae (Coleoptera) de zonas áridas del sureste de la Península Ibérica. Boletim da Sociedade Portuguesa de Entomologia, 2 (suplemento 3) (Actas V Congresso Ibérico de Entomologia) [1992]: 325-335.

Soumakov, G.G., 1915. Les espèces paléartiques du genre Mylabris Fabr. (Coleoptera, Meloidae). Horae Societatis Entomologicae Rossicae, 42: 1-71.

Soumakov, G.G., 1934. Inventa entomologica itineris hispanici et maroccani quod a 1926 fecerunt Harald et Hakan Lindberg. XXI. Méloides du Bassin Méditerranéen et du Maroc. Commentationes Biologicae. Societas Scientiarum Fennica, 4(13): 1-6.

VAlladARES, L.F., 1984. Estudio de los coleópteros vesicantes de la provincia de León (Coleoptera, Meloidae). Institución "Fray Bernardino de Sahagún". Diputación Provincial de León, C.S.I.C. León. 156 pp. 
Apéndice I.- Material examinado. Las siglas utilizadas y su significado son los siguientes: MNCN: Museo Nacional de Ciencias Naturales (Madrid); IRSNB: Institut Royal des Sciences Naturelles de Belgique (Bruselas); JLR: colección J.L. Ruiz (Ceuta). Algunos especímenes no pudieron ser sexados, indicándose en este caso únicamente el número de ejemplares estudiados.

Appendix I.- Material examined. The acronyms used and its meaning are the following: MNCN: Museo Nacional de Ciencias Naturales (Madrid); IRSNB: Institut Royal des Sciences Naturelles de Belgique (Bruselas); JLR: collection J.L. Ruiz (Ceuta). In some specimens the sex could not be determined, showing in this case only the number of specimens studied.

Mylabris (Mesosulcata) thamii Kocher, 1963

MARRUECOS: Tizi-n-Tinifift, Agdz, 1600 m.s.n.m., 11-IV1990, G. Bastazo y J.M. Vela leg.: 60 $0^{\pi}$, 3 우 우 (MNCN y JLR).

Mylabris (Mesosulcata) hirtipennis Raffray, 1873

MARRUECOS: Tánger: 1 ejemplar (IRSNB); Glaui: 5 ejemplares (MNCN); Marraquesh, III-1907, M. Escalera leg.: 2 ejemplares (MNCN); Marraquesh, IV-1907, M. Escalera leg.: 4 ejemplares (MNCN); Marraquesh, V-1907, M. Escalera leg.:
5 ejemplares (MNCN); Mogador, V-1907, M. Escalera leg.: 2 ejemplares (MNCN); Mogador, VII-1905: 1 ejemplar (MNCN); Imlil, Tizi-n-Mizic, Alto Atlas, 7-VII-84, A. Tinaut leg.: $30^{\prime} \sigma^{x}$, 3 우 $ᄋ$ (JLR); Sidi Bou Outhmane, Crrta. Casablanca-Marrakech, 28-III-88, J.M. Vela y G. Bastazo leg.: $30^{7} \sigma^{7}, 1$ ㅇ (JLR); Puerto de Gurda, Ketama, Rif central, 4-VI90, J.M. Ávila leg.: $2 \sigma^{7} \sigma^{7}, 1$ O (JLR); Col du Zac, 2200 m.s.n.m., Atlas Medio, 28-VI-92, J.M. Ávila leg.: $20^{\pi} \sigma^{\pi}$ (JLR); Ajdir, Atlas Medio, 29-VI-95, F. Delgado leg.: 1 ㅇ (JLR); Safi, 11-IV-92, J.L. Ruiz leg.: $10^{7}$ (JLR). 